\title{
Two sources of task prioritization: The interplay of effector-based and task order-based capacity allocation in the PRP paradigm
}

\author{
Mareike A. Hoffmann ${ }^{1}$ (D) Aleks Pieczykolan ${ }^{2} \cdot$ Iring Koch $^{3} \cdot$ Lynn Huestegge $^{1}$ \\ Published online: 12 June 2020 \\ (C) The Author(s) 2020
}

\begin{abstract}
When processing of two tasks overlaps, performance is known to suffer. In the well-established psychological refractory period (PRP) paradigm, tasks are triggered by two stimuli with a short temporal delay (stimulus onset asynchrony; SOA), thereby allowing control of the degree of task overlap. A decrease of the SOA reliably yields longer RTs of the task associated with the second stimulus (Task 2) while performance in the other task (Task 1) remains largely unaffected. This Task 2-specific SOA effect is usually interpreted in terms of central capacity limitations. Particularly, it has been assumed that response selection in Task 2 is delayed due to the allocation of less capacity until this process has been completed in Task 1. Recently, another important factor determining task prioritization has been proposed — namely, the particular effector systems associated with tasks. Here, we study both sources of task prioritization simultaneously by systematically combining three different effector systems (pairwise combinations of oculomotor, vocal, and manual responses) in the PRP paradigm. Specifically, we asked whether task order-based task prioritization (SOA effect) is modulated as a function of Task 2 effector system. The results indicate a modulation of SOA effects when the same (oculomotor) Task 1 is combined with a vocal versus a manual Task 2 . This is incompatible with the assumption that SOA effects are solely determined by Task 1 response selection duration. Instead, they support the view that dual-task processing bottlenecks are resolved by establishing a capacity allocation scheme fed by multiple input factors, including attentional weights associated with particular effector systems.
\end{abstract}

Keywords Cognitive and attentional control $\cdot$ Dual-task performance $\cdot$ Dual task procedures (PRP)

\section{Introduction}

In everyday life, we are often confronted with situations in which we have to perform more than one task at a time. Typically, performance (in at least one of the tasks) suffers in such situations when compared with executing only one task in isolation (see Koch, Poljac, Müller, \& Kiesel, 2018; Pashler, 1994, for reviews). The aim of the present study was

This work was supported by Deutsche Forschungsgemeinschaft (German Research Foundation), HU 1847/4-1

Mareike A. Hoffmann

mareike.a.hoffmann@gmail.com

1 Institute of Psychology, University of Würzburg, Röntgenring 11, 97070 Würzburg, Germany

2 Human Technology Center, RWTH Aachen University, Aachen, Germany

3 Institute of Psychology, RWTH Aachen University, Aachen, Germany to systematically look at two factors that determine the priority with which tasks are processed in such dual-task situationsnamely, task order and the particular effector systems associated with tasks. Therefore, we combined tasks requiring oculomotor, vocal, and manual responses in pairwise combinations initiated with a short temporal delay in order to answer the question of whether task order-based task prioritization can be modulated by Task 2 effector system.

\section{Empirical approaches to dual tasking}

Several methodological approaches have been established to address cognitive mechanisms underlying dual-task control. For example, in continuous task paradigms, participants are trained to perform two or more tasks continuously over a longer time span (e.g., Peterson, 1969; Spelke, Hirst, \& Neisser, 1976; see also Künstler et al., 2018). Other research paradigms aimed at maximizing control over stimulus conditions and response execution by involving clearly defined sets of stimuli and responses with discrete onsets. 
One example is the simultaneous (stimulus) onset paradigm, in which two stimuli (each requiring a distinct single response) are presented at exactly the same time. Performance is then compared between single-task versus dual-task conditions (e.g., Hoffmann, Pieczykolan, Koch, \& Huestegge, 2019; Hoffmann, Westermann, Pieczykolan, \& Huestegge, in press; Huestegge \& Koch, 2013; Pieczykolan \& Huestegge, 2014, 2017, 2018; Schumacher et al., 2001; Stelzel, Schumacher, Schubert, \& D'Esposito, 2006). One disadvantage of this paradigm is that single-task and dualtask conditions differ with respect not only to the degree to which tasks are performed simultaneously but also whether one or two task representations need to be kept active during a particular trial. Thereby, in this paradigm it is difficult to exactly pinpoint the sources of any dual-task performance decrements (e.g., whether dual-task costs are caused by the actual motor demands in both tasks or whether mere preparation in terms of active mental representations of the two competing task sets is sufficient to yield performance decrements).

As a consequence, the probably most prominent paradigm to study dual-task processes is the psychological refractory period (PRP) paradigm, which involves the execution of two tasks in each trial, but with varying temporal overlap. Specifically, stimuli are presented with a short, variable temporal interval (stimulus onset asynchrony; SOA), which allows the researcher to systematically manipulate the degree of temporal overlap between cognitive requirements of the two tasks (Telford, 1931; Welford, 1952; see also Bratzke et al., 2008; Hirsch, Declerck, \& Koch, 2015; Janczyk, Augst, \& Kunde, 2014; Kunde, Wirth, \& Janczyk, 2018; Pashler, 1984, 1994; Strobach, Becker, Schubert, \& Kühn, 2015). A well-established finding is that a decrease of SOA yields an increase in reaction times (RTs) of Task 2 (i.e., the task in which the stimulus was presented second and in which the response is usually executed second), whereas Task 1 RTs typically remain unaffected by SOA manipulations. This pattern of SOA effects is typically referred to as the PRP effect (e.g., Bratzke et al., 2008; Fagot \& Pashler, 1992; Janczyk \& Kunde, 2010; Pashler, 1984; see also Pashler, 1994). Note that the term "PRP effect" already implies a priori the existence of a refractory period, in which no parallel processing can take place (cf. also Navon \& Miller, 2002). As we want to avoid such theoretical connotations regarding the present study, we will simply refer to this predicted pattern as the "SOA effect."

\section{The response selection bottleneck model}

Pashler (1994) interpreted this SOA effect in dual-task performance by assuming that the central processing stages of the two tasks (i.e., the stage at which the task-appropriate response has to be selected) can only occur serially (response selection bottleneck [RSB] model), whereas stimulus processing and response execution of one task were assumed to be processed in parallel to any stage of the other task. This central bottleneck is conceptualized in an all-or-none fashion based on task order: Response selection in Task 2 cannot proceed until response selection in Task 1 is finished. This interruption of Task 2 processing has also been referred to as "slack time" (Schweickert, 1978). Note that according to this view, the SOA effect (in Task 2 RTs) is solely determined by the duration of response selection in Task 1 . The main reason for the bottleneck-based prioritization of Task 1 over Task 2 is that stimulus processing in Task 1 is usually finished first (due to SOA), and thereby Task 1 arrives earlier at the central (response selection) stage.

Interestingly, an SOA effect was also demonstrated using different effector systems, including oculomotor responses (e.g., Pashler, Carrier, \& Hoffman, 1993). Later, Bratzke et al. (2008) additionally demonstrated the stability of the SOA effect when the effector systems were reversed across tasks. Note, however, that this study did not explicitly focus on a comparison of SOA effect size as a function of effector system reversal across the two tasks.

\section{Parallel central processing models}

However, the assumption of strictly serial central processing of response-related task features has been challenged on various levels (see Pieczykolan \& Huestegge, 2019; see also Koch et al., 2018, for a recent review). Hommel (1998), for example, proposed the presence of partially parallel central processing of response-related features across tasks. Particularly, his data suggested that response selection of Task 1 is affected by response-related features of Task 2 . Specifically, Task 1 performance was influenced by the response-response compatibility relation between the two tasks (as well as by the compatibility between the secondary response and the primary stimulus), a phenomenon referred to as backward crosstalk/compatibility effect (Hommel, 1998; see also Durst \& Janczyk, 2018, 2019; Hommel \& Eglau, 2002; Huestegge, Pieczykolan, \& Janczyk, 2018; Janczyk, Pfister, Hommel, \& Kunde, 2014; Janczyk \& Huestegge, 2017; Janczyk, Renas, \& Durst, 2018; Renas, Durst, \& Janczyk, 2018). This suggests that at least some aspect of response-related central processing (e.g., response activation) can occur in parallel.

Moreover, there are also theories that go a step further and assume that completely parallel response selection across tasks is principally possible, and that during dual tasking, limited cognitive capacity is continuously shared among (and differentially allocated to) tasks (e.g., based on strategic reasons; Meyer \& Kieras, 1997; Navon \& Miller, 2002; Tombu \& Jolicœur, 2003). In fact, these models make predictions quite similar to a serial response selection account. For 
example, they assume that a major portion of capacity is allocated to Task 1 response selection first until capacity is later shifted (in either a gradual or step-wise manner) to complete Task 2 response selection. Thus, instead of an "inflexible", allor-none allocation mechanism (as suggested by the term "refractory" in the term "psychological refractory period effect") based on subtask order, these more recent theories allow for flexible, graded capacity sharing that can be modulated by particular task demands or instructions (see Fischer \& Plessow, 2015, for an overview; Logan \& Gordon, 2001, for a discussion; cf. also Huestegge \& Koch, 2010, for a crossmodal crosstalk model).

Consequently, Navon and Miller (2002) as well as Tombu and Jolicœur (2003) argued that central capacity sharing models can yield the same predictions as a structural all-ornone bottleneck model, and that the latter can be seen as a special variant of capacity sharing. Evidence for flexible capacity allocation comes, for example, from a study by Miller, Ulrich, and Rolke (2009), who observed more parallel processing in conditions where short SOAs were particularly frequent. Taken together, capacity sharing models interpret SOA effects in terms of a reflection of a particular capacity allocation scheme (usually prioritizing Task 1 ), which can, however, principally be modulated by task characteristics.

\section{The role of effector systems in dual tasking}

A different line of studies recently identified another potential factor influencing capacity allocation in multiple (simultaneous) action control situations - namely, the particular effector systems associated with the tasks at hand. A study by Huestegge and Koch (2013) on single versus dual actions triggered by single stimuli demonstrated evidence for flexible, effector-based capacity allocation between simultaneously triggered actions in different effector systems as indexed by the size of corresponding dual-action costs (defined as the RT difference between single-action and dualaction performance). More specifically, the relative size of dual-action costs suggested a prioritization of oculomotor responses (smallest dual-action costs) over vocal responses, and of vocal over manual responses (largest dual-action costs).

Recently, we demonstrated that similar effects can also be observed in another dual-task setting - namely, in the simultaneous onset paradigm involving two stimuli that independently triggered two responses (Hoffmann et al., 2019). In this particular study, we replicated the ordinal prioritization pattern referred to above (additionally including foot responses, which were prioritized over both vocal and manual responses).

In the dual-task context, these results cannot be explained by any "first come, first served" mechanism, since the effector-based prioritization (i.e., the size of dual-task costs) was not ranked in accordance with absolute task RT levels (cf. also Pieczykolan \& Huestegge, 2014, 2018). Note that such a "first come, first served" mechanism is not only a part of basic RSB models of response scheduling, but also (at least implicitly) of more recent flexible capacity scheduling models. That is, even without assuming strictly serial response selection, flexible capacity sharing models also need to provide an answer to the question of how capacity is allocated among tasks, which is usually referred to stimulus sequence (or task processing speed; see, e.g., Logan \& Gordon, 2001).

\section{Factors affecting capacity allocation}

The findings described above once again highlight that the observation of response selection queuing in PRP studies is a rather special case of capacity allocation that is brought about by sequential stimulus presentation. However, several other factors were proposed that influence capacity scheduling. For example, there is already evidence that factors such as SOA distribution (e.g., Miller et al., 2009), the proportion of congruent (vs. incongruent) trials (e.g., Fischer, Gottschalk, \& Dreisbach, 2014) or instructions (e.g., Lehle \& Hübner, 2009) can influence how response selection capacity is shared.

Another form of capacity allocation has been discussed in the context of superordinate processes responsible for implementing task order in the form of task order sets (cf. De Jong, 1995; Hirsch, Nolden, \& Koch, 2017; Hirsch, Nolden, Philipp, \& Koch, 2018; Kübler, Reimer, Strobach, \& Schubert, 2018; Luria \& Meiran, 2003). Specifically, it is assumed that the two subtasks are not represented independently, but grouped as one single higher-order task that integrated both the individual subtask task sets and task order information. This assumption is, for instance, supported by the observation of task set switch costs (cf. Hirsch et al., 2017; Hirsch et al., 2018) as well as specific costs yielded by task order alternations (cf. e.g., Kübler et al., 2018; Luria $\&$ Meiran, 2003) or recent studies on task order control with endogenously prepared and predictable task order information (Strobach, Kübler, \& Schubert, 2019).

Among all these relevant sources of influence for capacity scheduling reviewed so far, the present study is mainly focused on effector systems as highly influential factors that determine weighting mechanisms when allocating capacity to the individual response selection processes. Specifically, during parallel processing of tasks executed with different effector systems, we assume that effector-related characteristics become already functionally effective (i.e., anticipated) at a relatively early stage of task processing. Based on this, capacity is shared unevenly among tasks in that more capacity is shifted towards tasks associated with effector systems that are located higher up on an ordinal hierarchy (e.g., prioritization 
of oculomotor tasks over tasks involving other effector systems; cf. Hoffmann et al., 2019).

\section{The interplay of task order and effector systems}

Therefore, both task order-related processes relevant for typical SOA effects in the PRP paradigm and effector systemrelated prioritization represent two sources of influence that evidently affect task prioritization in terms of capacity allocation in dual-task control. So far, however, the interplay between these two sources of task prioritization remained elusive. In particular, flexible capacity sharing accounts, which embrace the view that SOA effects are a reflection of flexible and parallel central capacity allocation, principally allow for modulations of the SOA effect on Task 2 performance. These modulations might come, for example, from effector systems associated with the tasks. Previous dual-task studies that focused on SOA effects using different effector systems did not explicitly tackle this potential modulation of the SOA effect under otherwise controlled conditions (e.g., Bratzke et al., 2008; Fagot \& Pashler, 1992; Hibberd, Jamson, \& Carsten, 2010; Janczyk \& Kunde, 2010).

Two outcomes are conceivable: On the one hand, typical task order-based effects such as the SOA effect might be so strong that the corresponding allocation policy is immune to any effects related to effector systems. Here, we refer to the resulting temporal sequence of assigning major portions of capacity to the two tasks at the level of response selection. This would be plausible under the assumption that effector system information comes into play relatively late during task processing (e.g., only at the last stage of response executionrelated processing). However, on the other hand, it is also possible that participants anticipate specific characteristics of the effector systems associated with the two tasks (e.g., anticipated proximal sensory response effects; cf. e.g., Pfister, Janczyk, Gressmann, Fournier, \& Kunde, 2014; Pfister \& Kunde, 2013) so that effector system prioritization mechanisms might modulate the capacity allocation scheme. The latter assumption would predict that the SOA effect on Task 2 performance in the context of the same Task 1 might vary as a function of the effector system associated with Task 2.

\section{The present study}

To address this question, we combined oculomotor, vocal, and manual responses in three pairwise combination groups in a PRP setup involving counterbalanced auditory and visual stimuli and spatial left/right responses for the two tasks. Within these combination groups, we manipulated task order so that each effector system served as Task 1 or as Task 2 in different blocks.

We generally expected to observe higher Task 2 RTs under short SOAs than under long SOAs for all effector systems (i.e., typical influence of SOA on RT). Note that we did not focus on comparing SOA effects as a function of effector system order within each pairwise group of effector systems. Any significant effects of such a comparison would be confounded by the fact of a different effector system in Task 1 , and could therefore not conclusively demonstrate prioritization processes among effector systems. For example, differences in the duration of an effector-specific response selection stage between the two Task 1s could also lead to asymmetrical SOA effects without providing clear evidence for an influence of Task 2 effector system. Instead, we compared SOA effects on Task 2 performance across pairwise groups - specifically, in dual-task settings with different Task 2 effector systems, but constant Task 1 effector systems (e.g., comparison of vocal and manual Task 2 performance that are combined with the same oculomotor Task 1).

If the SOA effect in Task 2 is mainly determined by Task 1 response selection duration (e.g., as one would expect within a classic all-or-none RSB framework), the SOA effect observed in Task 2 RTs in the context of the same Task 1 should be independent of the effector system associated with Task 2 . However, if the SOA effect reflects principally flexible capacity allocation (prioritization of Task 1), and if effector systems impact on the capacity allocation scheme in dual tasking, then this allocation (reflected in the size of the SOA effect) could be modulated by the effector system associated with Task 2 . Specifically, the SOA effect (as a marker of the extent to which Task 1 is prioritized over Task 2) could be smaller when Task 2 involves responses executed by effector systems with a higher priority within the ordinal effector-based prioritization pattern.

Therefore, we compared Task 2 performance as a function of the effector system of Task 2 while the effector system of Task 1 was held constant. This resulted in three separate mixed comparisons with the within-subject factor SOA and the between-subject factor group (ultimately referring to Task 2 effector system). More specifically, we compared the influence of SOA on Task 2 performance for vocal versus manual responses after an oculomotor Task 1, on oculomotor versus vocal Task 2 performance after a manual Task 1, and on oculomotor versus manual Task 2 performance after a vocal Task 1 (see Table 1 for further illustration of this logic).

\section{Method}

\section{Participants}

We tested 24 participants in each of the three pairwise combination groups of oculomotor, vocal, and manual left/right 
Table 1 Overview of the three main comparisons, highlighting which effector system is associated with Task 1 (T1) and Task 2 (T2), respectively

\begin{tabular}{lcccc}
\hline & Constant T1 & Conditions to be compared & \\
\hline Comparison 1 & Oculomotor T1 & Vocal T2 & vs. & Manual T2 \\
Comparison 2 & Manual T1 & (of oculomotor-vocal group) & (of oculomotor-manual group) \\
& Vocal T1 & Oculomotor T2 & Vocal T2 \\
Comparison 3 & & (of oculomotor-manual group) & (of vocal-manual group) \\
& & Oculomotor T2 vs. & Manual T2 \\
(of oculomotor-vocal group) & (of vocal-manual group) \\
\hline
\end{tabular}

Note. Comparisons imply between-subject comparisons. Task order within each effector system combination pairing was manipulated within subjects

tasks, resulting in a total of 72 participants. Across all three groups, 14 participants had to be excluded because their data contained too many invalid or erroneous (above a criterion of $33 \%$ ) trials, or due to technical issues during data collection (three participants). We recollected these data (14 new participants) to ensure full counterbalancing and constant group size. Twenty-two out of the final 72 participants were male, and the mean age was 24.3 years $(S D=6.6)$. All were naïve regarding the purpose of the study and gave informed consent. Participants were recruited from the local university's student panel and received monetary reward or course credit for participation. They were all right-handed and had normal or corrected-to-normal hearing and vision.

\section{Apparatus and stimuli}

Participants were seated approx. $67 \mathrm{~cm}$ in front of a 21-inch cathode ray tube screen. Spatial resolution was $1,024 \times 768$ pixels, with a temporal resolution of $100 \mathrm{~Hz}$. An eye tracker sampling eye movements at $1000 \mathrm{~Hz}$ (EyeLink 1000, SR Research Mississauga, Ontario, Canada) was used to register saccade latencies and direction (right eye) in conditions requiring oculomotor responses, and to control for unintended saccades in the vocal-manual combination group (in which oculomotor responses were not required). Head movements were minimized by using a chin rest. When manual responses were required, participants should operate the left and right arrow key on a standard (German) QWERTZ keyboard unimanually with their right index finger, using the arrow down key as a home key. Vocal responses were registered by a microphone (Sennheiser e 835-S) in front of them. The software Experiment Builder (Version 2.1.140, SR Research) was used to run the experiment and to $\log$ the event times of oculomotor, manual, and vocal responses. The latter was measured by means of the integrated voice key function of the programming software.

Auditory stimuli consisted of $1000 \mathrm{~Hz}$ sinusoidal tones presented either to the left or right ear via supra-aural headphones. Visual stimuli consisted of easily distinguishable green arrows presented $0.43^{\circ}$ above the fixation cross pointing either to the left or to the right ("<" or " $>$ "; size $=0.86^{\circ}$ ).
Moreover, throughout each block, a green fixation cross (size $=0.43^{\circ}$ of visual angle) at the center and two green rectangular squares at an eccentricity of 8.5 degrees of visual angle $($ size $=$ $0.43^{\circ}$ each) to the left and right of the central fixation cross remained present on the screen (black background). These green rectangular squares served as targets for oculomotor responses (when required) but were also presented in the vocal-manual group to keep the visual input constant and to ensure comparability among groups and conditions.

\section{Procedure}

At the beginning of the experiment participants received instructions both verbally by a research assistant as well as visually on an instruction screen. The experiment consisted of four different block types. These block types were based on two task orders (e.g., vocal response first, manual response second vs. manual response first, vocal response second in the vocal-manual combination group), and, for counterbalancing reasons, two stimulus modalities and thus two stimulus to response (S-R) modality mappings. Note that in all three combination groups both possible assignments of stimulus modalities to effector systems were implemented (e.g., visual stimuli combined with manual responses and auditory stimuli combined with vocal responses; i.e., VM-AV, vs. the opposite pairing, VV-AM, VO-AM vs. VM-AO, and $\mathrm{VO}-\mathrm{AV}$ vs. $\mathrm{VV}-\mathrm{AO})$ to control for any influence of the assignment of stimulus modality and effector systems. In total, there were eight blocks (64 trials each) with a total duration of approximately 45 minutes. The $\mathrm{S}-\mathrm{R}$ modality mapping stayed consistent for the first half of the experiment and was changed after the first half of the experiment (order of S-R modality mappings was counterbalanced across participants). The sequence of the different task-order blocks was also counterbalanced across participants (but kept constant for each individual).

Stimuli were always presented in the order in which participants were required to respond to them. For example, in the "manual first" condition of the vocal-manual combination group, participants in the VM-AV mapping condition always 
saw the visual stimulus prior to hearing the auditory stimulus. Each individual block started with an instruction regarding stimulus order, corresponding required response order, and the particular S-R modality mapping.

Instructions were followed by a three-point horizontal calibration routine. Both stimuli (visual arrow and auditory sinusoidal tone) were presented for $80 \mathrm{~ms}$ with a variable SOA of $50,200,400$, or $800 \mathrm{~ms}$. All directional combinations of both stimuli (arrow pointing to left or right and auditory signal on the left or right ear) occurred equally often under each experimental condition in randomized order.

Participants were instructed to focus on the central fixation cross throughout each block in the vocal-manual combination group, in which oculomotor responses were not required. In groups requiring an oculomotor response (oculomotor-vocal combination group and oculomotor-manual combination group), participants were instructed to execute a saccade to either the left or the right rectangular target square (both were presented at constant eccentricity from the fixation cross) and to return with their gaze to the fixation cross afterwards. When manual responses were required, participants were asked to position their right index finger loosely (without pressing the key) on the arrow down key on the keyboard, which was located centrally between the two response keys (arrow right and arrow left). Participants were instructed to return to the central key position after response execution. This was done to increase comparability to oculomotor responses that also consist of a movement from a starting position to a target position. Vocal responses consisted of uttering the words "links" or "rechts" (German for "left"/"right"). Responses should always be spatially congruent to the respective stimuli.

The first of the two stimuli were presented with a fixed interstimulus interval (ISI) of 3,000 ms between two consecutive trials. If participants responded in the wrong order (as defined by instructions and stimulus order), or if they responded with one of the two effector systems before the corresponding stimulus had been presented (e.g., execution of a saccade prior to onset of the visual stimulus in the VOAM S-R modality mapping), an error feedback was presented (300 ms, either "error" or "to soon" plus an additional reminder of the respective task requirements, e.g., "key press first" or "saccade first" in the context of a "wrong order" feedback and "pay attention to the tone/arrow" in the context of premature responses).

\section{Design}

The within-subjects factor was SOA $(50 \mathrm{~ms}, 200 \mathrm{~ms}, 400 \mathrm{~ms}$, $800 \mathrm{~ms}$ ) and the between-subjects factor was group (representing the effector system of Task 2: oculomotor, vocal, or manual, combined with the respective other effector systems in Task 1) in a mixed design. Note that we controlled for the influence of stimulus modality of Task 2 by instantiating both S-R modality mapping conditions (in counterbalanced order). For the sake of conciseness, we report results averaged over the two S-R modality mapping conditions, but we additionally provide results of separate analyses for all S-R modality mappings in Tables 2 and 3 in the Appendix. RTs and error rates (ERs) served as dependent variables. Crucial comparisons were those related to the interaction of SOA and group for the same Task 1 effector system condition. That is, in three (overlapping), pairwise mixed $4(\mathrm{SOA}) \times 2$ (group; i.e., Task 2 effector system) analyses of variance (ANOVAs), we compared SOA effects on Task 2 performance in the oculomotor-vocal versus oculomotor-manual group when the oculomotor response was associated with Task 1 (Analysis 1), on Task 2 performance in the oculomotor-manual versus vocal-manual group when the manual response was associated with Task 1 (Analysis 2), and on Task 2 performance in the oculomotor-vocal versus vocalmanual group when the vocal response was associated with Task 1 (Analysis 3; see Table 1 for a further illustration of this logic).

\section{Results}

In case of sphericity violations, Greenhouse-Geisser corrected degrees of freedom and statistics are presented in all analyses.

\section{Data treatment}

Of all trials, $8.5 \%$ were excluded from further analysis because they contained an omission error regarding at least one of the required responses. Further, $2.5 \%$ of trials included responses executed in the wrong order, and another $1.4 \%$ were excluded because an unwarranted saccade was registered (prior to the actually required responses) in conditions that did not require saccades. Note that omission errors were not considered in the error analysis because of effector-specific baseline differences in the occurrence of such omissions - for example, based on sensitivity differences of the devices that capture the various responses in the different effector systems.

Next, responses given within the first $50 \mathrm{~ms}$ after stimulus onset were discarded (further $0.3 \%$ ), for example, to ensure the exclusion of voice key artefacts. Further, outliers were defined as responses slower or faster plus/ minus two standard deviations from the individuals mean in each condition. These were also excluded (6.8\%). In $6.0 \%$ of the remaining valid trials, a directional error (e.g., right instead of left) in at least one of the two responses was made. Only valid and correct trials were considered in the RT analyses. 


\section{Reaction times}

Figure 1 shows that typical SOA effects (in terms of an increase of Task 2 RT with decreasing SOA) were observed throughout all groups and conditions. This indicates a general prioritization of Task 1 (i.e., due to instructed task order), in line with our predictions, and represents a necessary prerequisite for any search for modulations of this effect by Task 2 effector system.

As outlined above, we were interested in differences between the SOA effects for a constant Task 1 (in terms of its associated effector system and controlled for stimulus modality) as a function of different effector systems in Task 2 (i.e., for the two lines representing Task 2 performance within each

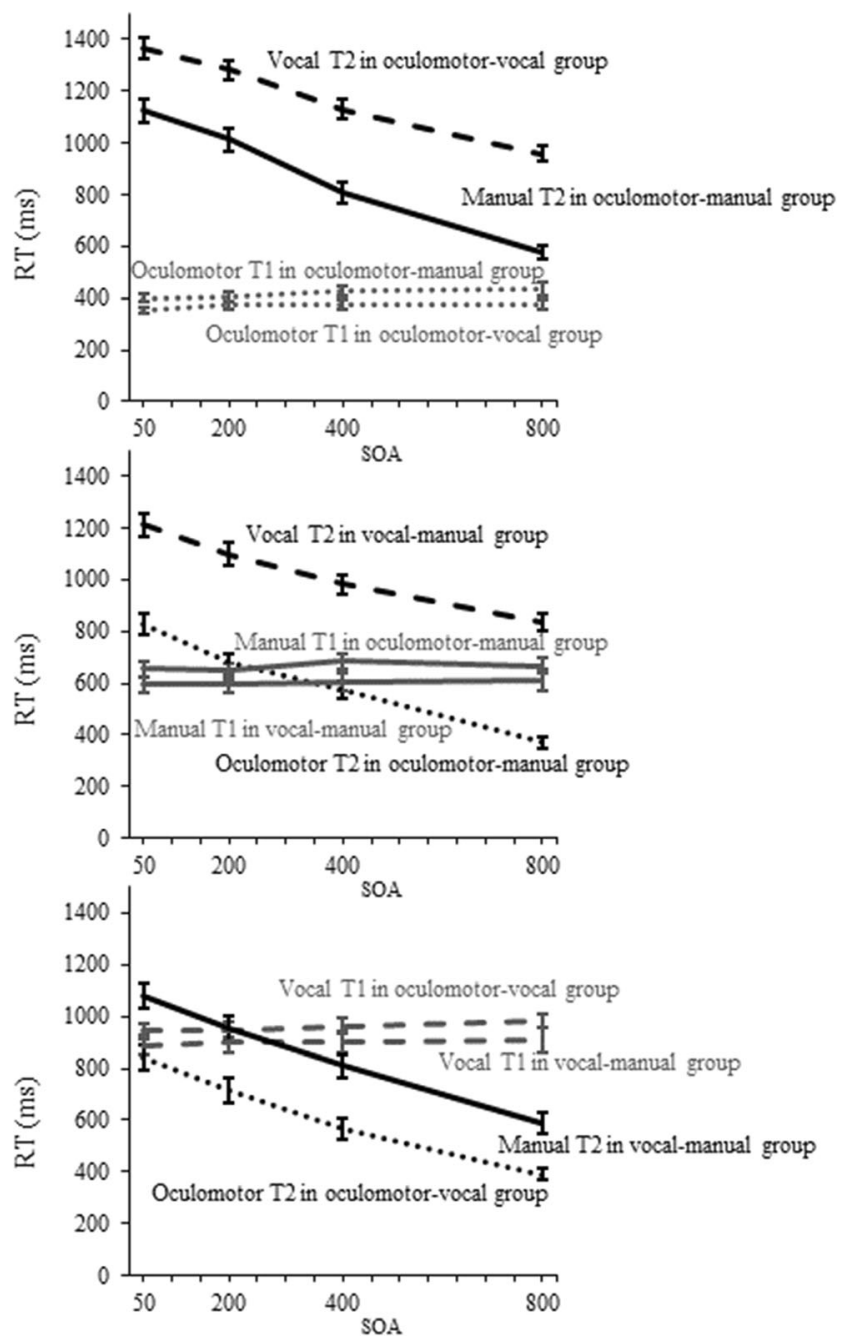

Fig. 1 RTs for Task 1 and Task 2 for the three main comparisons. Compared conditions (i.e., those involving the same Task 1, but different Task 2 effector systems) are presented within one panel: vocal versus manual Task 2 with constant oculomotor Task 1 in the upper panel, oculomotor versus vocal Task 2 with constant manual Task 1 in the middle panel, and oculomotor versus manual Task 2 with constant vocal Task 1 at the bottom. Error bars represent (unadjusted) standard errors of the individual means $(S E M)$. SOA = stimulus onset asynchrony graphs in Fig. 1). This resulted in three $2 \times 4$ ANOVAs, with the between-subjects independent variable group (Task 2 effector system) and the within-subjects independent variable SOA. ${ }^{1}$

For the comparison of vocal and manual Task 2 responses after an oculomotor response in Task 1 (see top panel of Fig. 1), we observed a significant main effect of group: Manual responses $(881 \mathrm{~ms})$ were overall faster than vocal responses $(1,184 \mathrm{~ms}), F(1,46)=33.65, p<.001, \eta_{\mathrm{p}}^{2}=.42$. The main effect of SOA, representing an increase of RTs with decreasing SOAs (768 ms vs. $970 \mathrm{~ms}$ vs. $1,147 \mathrm{~ms}$ vs. $1,245 \mathrm{~ms}$ ), $F(1.63,75.14)=554.32, p<.001, \eta_{\mathrm{p}}{ }^{2}=.92$, was also significant. Crucially, we observed a significant interaction of group and SOA, showing a stronger SOA effect for manual (RT difference between shortest and longest SOA: $547 \mathrm{~ms}$ ) than for vocal responses (RT difference between shortest and longest SOA: $409 \mathrm{~ms}), F(1.63,75.14)=11.78, p<.001, \eta_{\mathrm{p}}{ }^{2}=$ .20. Additionally, as can be seen in Table 2 in the Appendix, this effect was observed in each of the two individual $\mathrm{S}-\mathrm{R}$ modality mapping conditions (SOA effect: $514 \mathrm{~ms}$ vs. $349 \mathrm{~ms}$ for visually triggered Task 2 , and $590 \mathrm{~ms}$ vs. $488 \mathrm{~ms}$ for auditorily triggered Task 2).

For the comparison of oculomotor and vocal Task 2 RTs following a manual response in Task 1 (see middle panel of Fig. 1), we observed that oculomotor responses (613 ms) were significantly faster than vocal responses $(1,031 \mathrm{~ms}), F(1,46)$ $=70.89, p<.001, \eta_{\mathrm{p}}^{2}=.61$. Task 2 RTs increased with decreasing SOAs $(603 \mathrm{~ms} ; 776 \mathrm{~ms} ; 889 \mathrm{~ms} ; 1,019 \mathrm{~ms})$, $F(1.59,73.17)=376.80, p<.001, \eta_{\mathrm{p}}^{2}=.89$. The interaction of group and SOA missed the critical alpha level, $F(1.59$, 73.17) $=3.21, p=.057, \eta_{\mathrm{p}}{ }^{2}=.07$. However, it should be noted that the numerical trend in this comparison was in the direction of a slightly stronger SOA effect on oculomotor (RT difference between shortest and longest SOA: $455 \mathrm{~ms}$ ) than on vocal responses (RT difference between shortest and longest SOA: $377 \mathrm{~ms})$.

In comparison of oculomotor and manual Task 2 RTs following a vocal Task 1 (see bottom panel of Fig. 1), the ANOVA revealed overall faster oculomotor $(627 \mathrm{~ms})$ than manual responses $(857 \mathrm{~ms}), F(1,46)=14.86, p<.001, \eta_{\mathrm{p}}{ }^{2}$ $=.24$, and increasing Task 2 RTs with decreasing SOAs (488 $\mathrm{ms}, 686 \mathrm{~ms}, 835 \mathrm{~ms}, 960 \mathrm{~ms}), F(1.48,68.09)=556.08, p<$ $.001, \eta_{\mathrm{p}}{ }^{2}=.29$. There was no significant interaction between group and SOA, $F(1.48,68.09)=1.60, p=.213, \eta_{\mathrm{p}}{ }^{2}=.03$.

Finally, we conducted two further analyses to rule out two potential alternative explanations of the data. Specifically, under the assumption of parallel capacity sharing between tasks the observed difference between vocal and manual SOA

\footnotetext{
${ }^{1}$ Note that we also analyzed Task 1 performance as a function of Task 2 effector system. However, we observed no significant influence of Task 2 effector system, either on overall Task 1 RTs or regarding the dependency of Task 1 RTs on SOA (all $p s>.05$ ). This suggests that the particular effector system associated with Task 2 did not affect Task 1 processing.
} 
effects (after an oculomotor Task 1) might have also been caused by different durations of an effector-specific (vocal vs. manual) response selection process. If response selection for vocal responses generally takes less time than that of manual responses, this could also lead to a more pronounced manual SOA effect without necessarily indicating any prioritization (i.e., merely due to the shorter time span during which capacity had to be shared). Then, however, this should also hold true for vocal and manual Task 1 responses combined with an oculomotor Task 2 . Therefore, to ensure that the observed difference between vocal versus manual SOA effects was not solely based on such an effector-specific response selection duration, we additionally compared the oculomotor SOA effects as a function of Task 1 effector system. That is, in addition to the rationale depicted in Table 1, we now also compared Task 2 performance of the oculomotor-vocal versus oculomotor-manual combination groups when Task 2 was associated with the oculomotor effector system and Task 1 with the vocal versus manual system, respectively. The corresponding $4(\mathrm{SOA}) \times 2$ (group, i.e., now referring to different Task 1 effector systems) ANOVA revealed a significant effect of SOA, $F(1.50,69.18)=372.01, p<.001, \eta_{\mathrm{p}}{ }^{2}$ $=.89$, but, crucially, neither a significant effect of group, $F(1$, $46)=0.08, p=.777, \eta_{\mathrm{p}}{ }^{2}=.00$, nor a significant interaction, $F(1.50,69.18)=0.71, p=.547, \eta_{\mathrm{p}}{ }^{2}=.02$. Thus, we can rule out that the differences between vocal and manual SOA effects could be explained in terms of different durations of effector-specific vocal versus manual response selection processes.

Second, one could also argue that different effector-system combinations might go hand in hand with a different amount of cross-task interference based on spatial response-response incompatibility. For example, one might assume that more interference might occur between an oculomotor response to the "right" in Task 1 and an incompatible manual "left" key press in Task 2 than with a merely conceptually incompatible "left" vocal response in Task 2 (as oculomotor and manual responses are both physically spatial in nature). If so, it would not be surprising when asymmetric compatibility effects interact with SOA, as one would generally expect more interference at short SOAs. To rule out this alternative explanation for our finding that a stronger SOA effect occurred for manual than for vocal responses, we additionally compared spatial compatibility effects in manual versus vocal Task 2 performance after a (compatible vs. incompatible) oculomotor Task 1 and their interaction with SOA by conducting a corresponding $2 \times 2 \times 4$ ANOVA. Results revealed that responseresponse compatibility indeed affected Task 2 RTs, $F(1,46)$ $=12.31, p=.001, \eta_{\mathrm{p}}{ }^{2}=.21$, as there were faster Task 2 responses after a compatible $(1,023 \mathrm{~ms})$ than after an incompatible Task 1 (1,045 ms). Furthermore, this compatibility effect interacted with SOA, $F(3,138)=5.83, p=.001, \eta_{\mathrm{p}}{ }^{2}$ $=.11$, in that it decreased with increasing SOAs $(39 \mathrm{~ms}, 38$ $\mathrm{ms}, 9 \mathrm{~ms}, 0 \mathrm{~ms}$ from short to long SOA). Crucially however, the ANOVA revealed neither an interaction of compatibility and Task 2 effector system, $F(1,46)=0.27, p=.604, \eta_{\mathrm{p}}{ }^{2}=$ .01 , nor a three-way interaction of compatibility, Task 2 effector system, and SOA, $F(3,138)=0.56, p=.645, \eta_{\mathrm{p}}{ }^{2}=.01$, thereby ruling out asymmetric compatibility effects as a potential alternative explanation for the observed asymmetries in the SOA effect between vocal and manual Task 2 responses. In addition, note that our main result (smaller vocal than manual SOA effect) could be observed for both compatible and incompatible trials in corresponding separate analyses (see Table 4 in the Appendix).

\section{Error rates}

We conducted the same three ANOVAs for Task 2 error rates (ERs), even though errors occurred relatively rarely overall (6\% of valid data).

Regarding the comparison of Task 2 ERs between vocal versus manual Task 2s (after an oculomotor Task 1) ERs increased significantly with decreasing SOAs (1.5\%, 2.6\%, $3.1 \%, 5.1 \%), F(2.32,106.89)=12.64, p<.001, \eta_{\mathrm{p}}{ }^{2}=.22$, but did not differ significantly overall between the vocal $(2.6 \%)$ versus the manual $(3.3 \%)$ task, $F(1,46)=0.48, p=$ $.494, \eta_{\mathrm{p}}{ }^{2}=.01$. Unlike in RTs, there was no interaction of group and SOA regarding ERs, $F(2.32,106.89)=0.69, p=$ $.526, \eta_{\mathrm{p}}{ }^{2}=.02$.

Similarly, the comparison of vocal versus oculomotor Task 2 ERs (after a manual Task 1) revealed a significant effect of $\operatorname{SOA}(2.9 \%, 4.4 \%, 5.9 \%, 5.7 \%), F(3,138)=8.79, p<.001$, $\eta_{\mathrm{p}}{ }^{2}=.16$, but neither an effect of group (oculomotor: $4.9 \%$; vocal: $4.5 \%), F(1,46)=0.06, p=.803, \eta_{\mathrm{p}}{ }^{2}=.00$, nor an interaction, $F(3,138)=1.59, p=.194, \eta^{2} \eta_{\mathrm{p} p}^{2}=.03$.

Lastly, comparing oculomotor versus manual Task 2 ERs (after a vocal Task 1) revealed a significant effect of group (indicating higher ERs in oculomotor: $4.5 \%$ than in manual responses: $1.5 \%), F(1,46)=18.27, p<.001, \eta_{\mathrm{p}}{ }^{2}=.28$, but no effect for $\operatorname{SOA}(2.5 \%, 3.9 \%, 5.6 \%, 6.9 \%), F(2.26,104.04)=$ $1.77, p=.156, \eta_{\mathrm{p}}{ }^{2}=.04$, and no interaction, $F(2.26,104.04)=$ $1.36, p=.259, \eta_{\mathrm{p}}^{2}=.03$. $^{2}$

\footnotetext{
${ }^{2}$ It should be mentioned that when analyzing error rates for the two S-R modality mappings separately (see Table 3 in the Appendix), we did in fact observe a typical SOA effect when comparing visually triggered oculomotor and manual Task 2 ERs after an auditory-vocal Task 1 (2.5\%, 3.9\%, 5.6\%, $6.9 \%$ over decreasing SOAs). This was, however, cancelled out by an unexpected significant effect for SOA in the opposite direction in auditorily triggered oculomotor ERs after a visual-vocal Task 1 (SOA $800 \mathrm{~ms}: 4.3 \%$, SOA $400 \mathrm{~ms}: 3.7 \%$, SOA $200 \mathrm{~ms}: 2.1 \%$, SOA $50 \mathrm{~ms}: 0.7 \%$ ). Since there was no notable alteration of the generally low manual ERs among SOAs $(0.2 \%, 1.0 \%$, $0.8 \%, 0.5 \%$ ), this resulted in a significant effect of SOA overall (in an unusual pattern: $2.2 \%, 2.3 \%, 1.4 \%, 0.6 \%$ ) as well as in an interaction of SOA and group.
} 


\section{Discussion}

The aim of the present study was to test whether the effect of SOA on Task 2 performance (i.e., an increase of Task 2 RTs with decreasing SOA), which is typically interpreted as a reflection of strong initial capacity allocation to Task 1 response selection, can be modulated by the Task 2 effector system. Here, we assume that the SOA effect is regarded as indexing a flexible capacity allocation regime. Importantly, based on this reasoning, it is possible that the SOA effect can then be affected by effector systems associated with the tasks, we can expect a modulation of the SOA effect as a function of the effector system associated with Task 2 (cf. e.g., Hommel, 1998; Meyer \& Kieras, 1997; Navon \& Miller, 2002; Tombu \& Jolicœur, 2003), in line with the ordinal prioritization pattern regarding effector systems established in prior research (Hoffmann et al., 2019; Hoffmann et al., in press; Huestegge \& Koch, 2013; Pieczykolan \& Huestegge, 2014).

Here, we addressed this issue by systematically combining comparable PRP tasks (always involving simple left/right decisions based on left/right stimuli) that differed with respect to their associated effector systems (oculomotor, manual, vocal). All pairwise combinations of these effector systems were implemented across three groups of participants, while effector system (or task) order was manipulated and S-R modality mapping was controlled for within each group.

\section{Summary of main findings}

We observed the expected SOA effect (prolongation of Task 2 RTs with decreasing SOA) in all groups and conditions. The most important result here is that the SOA effect depended on the Task 2 effector system under constant Task 1 requirements (i.e., when Task 1 involved the same effector system), at least for the oculomotor Task 1. Specifically, following the oculomotor Task 1, the SOA effect was more pronounced when Task 2 involved a manual response compared with a vocal response (SOA effect, measured as the RT difference between the shortest and the longest SOA: $547 \mathrm{~ms}$ vs. $409 \mathrm{~ms}$ ).

This observation of an effector system-dependent SOA effect further strengthens the idea of flexible capacity sharing among tasks and speaks against a basic all-or-none response selection bottleneck model without major additional assumptions, as according to the latter the slack time responsible for the SOA effect (which is determined by Task 1 response selection duration) should be independent of Task 2 response characteristics. Further, it shows that effector systems are an influential factor in the decision process that determines how capacity is allocated among tasks: task prioritization is based not only on task order (as posited by both all-or-none and capacity sharing explanations of SOA effects) but also on specific effector system characteristics. Note that the interpretation of this effect was not compromised by the error data, because there was no counteracting effect in the error data regarding the crucial interaction of Task 2 effector system and SOA. Also note that this finding of vocal-over-manual prioritization corroborates findings from previous studies using other multitasking settings (e.g., Hoffmann et al., 2019; Hoffmann et al., in press; Huestegge \& Koch, 2013; Pieczykolan \& Huestegge, 2014).

\section{Potential alternative explanations}

At first sight, one might argue that such a modulation of the SOA effect by Task 2 effector system could also have resulted from effector system-specific (and thus unequally long) response selection processes in Task 2 (assuming the occurrence of parallel response selection processes in the first place), or from an asymmetry among Task 2 effector systems in the susceptibility to incompatible Task 1 information. However, the lack of any significant influence of Task 1 effector system on oculomotor Task 2 SOA effects and the finding of similar patterns for spatially compatible and incompatible trials render these explanations highly unlikely.

Finally, another general objection against our interpretation of the data might be to argue that the finding of smaller SOA effects for conditions involving a larger RT difference between Task 1 and Task 2 (as was the case for the vocal vs. manual Task 2 when combined with an oculomotor Task 1) should generally be expected, even based on most rudimentary response selection bottleneck models. At first sight, this might appear reasonable since large RT differences across tasks might decrease the potential for temporal overlap of response selection stages. However, it is crucial to bear in mind that we took great care in controlling stimulus-related processing duration in our relevant comparisons. Thereby, the end point of stimulus processing (which marks the crucial onset of the central bottleneck stage) is perfectly comparable, and thus there is no reason to assume a lower potential for central (response selection) stage overlap/competition for a vocal versus manual Task 2 given the same (oculomotor) Task 1. Crucially, Task 1 response selection duration (which should be the main determinant of any SOA effect in Task 2) should be perfectly comparable, and thus any basic bottleneck model would never predict differences in SOA effects based on different response-related Task 2 characteristics (i.e., differences in $\mathrm{T} 2$ effector system).

Consequently, our main finding can be interpreted as showing that task order-based task prioritization is affected by the effector systems involved in the tasks. Furthermore, the direction of the modulatory effect (larger SOA effect for manual Task 2) is consistent with the effector-based prioritization pattern assumed by Huestegge and Koch (2013; see also Hoffmann et al., 2019; Hoffmann et al., in press; Pieczykolan \& Huestegge, 2014), where it was suggested that vocal responses are generally prioritized over manual 
responses despite the fact that vocal responses are usually characterized by longer overall response latencies.

\section{Are oculomotor responses special?}

Our results show no significant differences in the SOA effect between the oculomotor versus vocal Task 2 following a manual Task 1, or between the oculomotor versus manual Task 2 following a vocal Task 1, although it should be mentioned that there was a trend towards a slightly higher oculomotor than vocal SOA effect in the former comparison. At first sight, the lack of a modulation of SOA effects in comparisons involving oculomotor Task 2 responses might appear to be at odds with the significant modulation of the SOA effect reported above (there was even a numerical trend towards oculomotor subordination instead of dominance), especially since previous research has provided strong evidence for a general prioritization of the oculomotor system over all other effector systems. However, if we take a closer look at the absolute oculomotor RT levels in comparison to manual and vocal Task 1 RTs (see Fig. 1), a reasonable explanation can be derived: Overall, oculomotor responses could be executed very quickly (about $395 \mathrm{~ms}$ as Task 1, compared with a Task $1 \mathrm{RT}$ range of 637-936 ms for vocal and manual responses). When comparing oculomotor and vocal (or manual) Task 2 performances (after a manual or vocal Task 1, respectively), it is quite likely that under short SOAs oculomotor responses were artificially withheld until the execution of the respective Task 1 response.

Note that this effect might have been further reinforced by our decision to provide error feedback in those trials in which participants responded in a reversed order than indicated by stimulus order. In future research, it might be of interest to investigate potential oculomotor dominance effects in the PRP paradigm when violations of task order are less prominently (or not at all) fed back to the participants. For example, Bratzke and colleagues (Bratzke, Rolke, Ulrich, \& Peters, 2007; Bratzke et al., 2008) repeatedly observed SOA effects even when participants were not explicitly instructed to execute responses in the order of corresponding stimulus presentation. However, such an approach could be challenging because a certain response order is a prerequisite of interpreting Task $2 \mathrm{RT}$ patterns in terms of an order-based SOA effect, and the few PRP studies involving oculomotor responses to date have shown that oculomotor responses are often executed first even when the corresponding stimulus was presented second (Pashler et al., 1993; Pieczykolan \& Huestegge, 2019). Thus, it might be possible that although oculomotor dominance (see Hoffmann et al., 2019; Huestegge \& Koch, 2013; Pieczykolan \& Huestegge, 2014) might also have an impact on cognitive processes in situations with sequential stimulation, a corresponding effect in the data may be difficult to demonstrate empirically.

\section{Theoretical implications}

Taken together, the present results indicate that task orderbased task prioritization (as typically assumed to explain SOA effects) can in fact be modulated by effector-based task prioritization. On a general level, these data are well in line with capacity sharing accounts of dual-task control (as, e.g., suggested by Tombu \& Jolicœur, 2003), which postulate that task processing can also run in parallel and does not have to strictly stick to an all-or-none capacity allocation scheme as suggested by classic bottleneck accounts. In addition, our results suggest that this specific allocation scheme-indicating which capacity portion is allocated to which task-depends on several task characteristics, including effector systems and their respective prioritization weight. However, note that the present study was not designed to ultimately decide between capacity sharing and (more complex variants of) all-or-none bottleneck models but rather to explore the degree to which capacity allocation might be influenced by effector-specific factors.

Potentially, this capacity allocation mechanism could be influenced by strategic factors in order to fulfill challenging task requirements in a time-limited setting as efficiently as possible. While in conditions involving sequential stimulus onset it provides strategic advantages to shift a lion's share of available capacity towards the task that was triggered first to get it "out of the way" and to reduce task interference, the extent of this task order-based task prioritization (as measured in the SOA effect on Task 2 RT) apparently can to some degree be adjusted as a function of more peripheral task characteristics.

The fact that certain task features which-according to traditional task processing logic - are usually regarded as "postcentral" (i.e., effector systems associated with the tasks) have such an effect on central capacity allocation policies do not seem to be consistent with basic assumptions of sequential task processing models. More specifically, these findings rather appear to support previous ideas that effector system representations are an integral part of central codes in working memory that need to be configured to eventually come up with task-appropriate action patterns. One model that considers this intuition was introduced by Huestegge and Koch (2010). Here, it was assumed that multiple-action control essentially requires the binding of task-relevant codes (e.g., spatial codes as well as codes specifying effector systems) in working memory (i.e., prior to action execution). Thus, according to this view, effector systems are an essential part of a central, complex code selection/binding process, and effector-related processing weights in such a model might be represented in terms of different connection strengths towards different effector systems. However, more research is needed to further substantiate the predictions of such a multiple action control model. 


\section{Conclusion}

In sum, we can conclude that effector system characteristicswhich, on a superficial level, might be assumed to exclusively influence relatively late processing stages - evoked substantial effects on dual-task control (Huestegge, 2011; Huestegge \& Adam, 2011; Huestegge \& Hazeltine, 2011; Huestegge, Pieczykolan, \& Koch, 2014). This highlights the importance of considering the particular effector systems involved in dual tasks when interpreting typical performance effects such as those resulting from classic SOA manipulations. Finally, the present study sheds further light on the specific mechanisms determining potential allocation regimes in capacity sharing models by indicating that several sources of influence, including task order and anticipatory processes regarding effector systems, are integrated to eventually determine a specific, demand-appropriate capacity allocation policy.

Funding Information Open Access funding provided by Projekt DEAL.

\section{Appendix}

Table 2. Overview of statistical test results of six separate ANOVAs for all possible specific Task 1 conditions regarding effector system and stimulus modality with RTs of Task 2 as the dependent variable, group (i.e., effector system of Task 2) as the independent between-subjects variable, and SOA as the independent within-subjects variable

\begin{tabular}{|c|c|c|c|c|c|c|c|c|}
\hline Source of variation & $d f$ & $F$ & $p$ & $\eta_{p}^{2}$ & $d f$ & $F$ & $p$ & $\eta_{\mathrm{p}}^{2}$ \\
\hline & \multicolumn{4}{|c|}{ Auditory-oculomotor Task 1} & \multicolumn{4}{|c|}{ Visual-oculomotor Task 1} \\
\hline Group (Manual vs. Vocal Task 2) & 1,46 & 29.70 & $<.001$ & .39 & 1,46 & 33.97 & $<.001$ & .43 \\
\hline SOA & $1.68,77.30$ & 365.44 & $<.001$ & .89 & $1.98,91.15$ & 443.22 & $<.001$ & .91 \\
\hline \multirow[t]{2}{*}{ Group $\times$ SOA } & $1.68,77.30$ & 13.64 & $<.001$ & .23 & $1.98,91.15$ & 4.46 & .014 & .09 \\
\hline & \multicolumn{4}{|c|}{ Auditory-manual Task 1} & \multicolumn{4}{|c|}{ Visual-manual Task 1} \\
\hline Group (Oculomotor vs. Vocal Task 2) & 1,46 & 61.97 & $<.001$ & .57 & 1,46 & 75.30 & $<.001$ & .62 \\
\hline SOA & $1.59,73.32$ & 190.03 & $<.001$ & .81 & $2.19,100.82$ & 350.62 & $<.001$ & .88 \\
\hline \multirow[t]{2}{*}{ Effector System $\times$ SOA } & $1.59,73.32$ & 1.38 & .255 & .03 & $2.19,100.82$ & 2.13 & .119 & .04 \\
\hline & \multicolumn{4}{|c|}{ Auditory-vocal Task 1} & \multicolumn{4}{|c|}{ Visual-vocal Task 1} \\
\hline Group (Oculomotor vs. Manual Task 2) & 1,46 & 10.81 & .002 & .19 & 1,46 & 14.49 & $<.001$ & .24 \\
\hline SOA & $2.05,94.38$ & 376.23 & $<.001$ & .89 & $1.60,73.79$ & 421.29 & $<.001$ & .92 \\
\hline Effector System $\times$ SOA & $2.19,94.38$ & 1.90 & .155 & .04 & $1.60,73.79$ & 2.07 & .143 & .04 \\
\hline
\end{tabular}

Table 3. Overview of statistical test results of six separate ANOVAs for all possible specific Task 1 conditions regarding effector system and stimulus modality with ERs in Task 2 as a dependent variable, group (i.e., effector system of Task 2) as an independent between-subjects variable, and SOA as an independent within-subjects variable

\begin{tabular}{|c|c|c|c|c|c|c|c|c|}
\hline Source of variation & $d f$ & $F$ & $p$ & $\eta_{\mathrm{p}}^{2}$ & $d f$ & $F$ & $p$ & $\eta_{\mathrm{p}}^{2}$ \\
\hline & \multicolumn{4}{|c|}{ Auditory-oculomotor Task 1} & \multicolumn{4}{|c|}{ Visual-oculomotor Task 1} \\
\hline Group (Manal vs. Vocal Task 2) & 1,46 & 3.04 & .088 & .06 & 1,46 & 0.42 & .520 & .01 \\
\hline SOA & $1.90,87.29$ & 10.39 & $<.001$ & .18 & $2.62,120.48$ & 2.77 & .052 & .06 \\
\hline \multirow[t]{2}{*}{ Group $\times$ SOA } & $1.90,87.29$ & 0.10 & .900 & .00 & $2.62,120.48$ & 1.78 & .161 & .04 \\
\hline & \multicolumn{4}{|c|}{ Auditory-manual Task 1} & \multicolumn{4}{|c|}{ Visual-manual Task 1} \\
\hline Group (Oculomotor vs. Vocal Task 2) & 1,46 & 1.78 & .189 & .04 & 1,46 & 0.29 & .591 & .01 \\
\hline SOA & $2.49,114.55$ & 7.83 & $<.001$ & .15 & $2.37,108.95$ & 3.38 & .020 & .07 \\
\hline \multirow[t]{2}{*}{ Effector System $\times$ SOA } & $2.49,114.55$ & 1.60 & .201 & .03 & $2.37,108.95$ & 0.74 & .531 & .02 \\
\hline & \multicolumn{4}{|c|}{ Auditory-vocal Task 1} & \multicolumn{4}{|c|}{ Visual-vocal Task 1} \\
\hline Group (Oculomotor vs. Manual Task 2) & 1,46 & 10.58 & .002 & .19 & 1,46 & 12.70 & .001 & .22 \\
\hline SOA & $2.27,104.18$ & 6.19 & .002 & .12 & 3,138 & 5.09 & .002 & .10 \\
\hline Effector System $\times$ SOA & $2.27,104.18$ & 0.29 & .776 & .01 & 3,138 & 5.93 & .001 & .11 \\
\hline
\end{tabular}


Table 4. Statistical test results of the supplementary analyses of Task 2 RT differences between manual versus vocal Task 2s after an oculomotor Task 1 presented separately for response-response compatible trials (left) versus response-response incompatible trials (right)

\begin{tabular}{|c|c|c|c|c|c|c|c|c|}
\hline \multirow[t]{2}{*}{ Source of variation } & \multicolumn{4}{|c|}{ Compatible trials only } & \multicolumn{4}{|c|}{ Incompatible trials only } \\
\hline & $d f$ & $F$ & $p$ & $\eta_{\mathrm{p}}^{2}$ & $d f$ & $F$ & $p$ & $\eta_{\mathrm{p}}^{2}$ \\
\hline Group (Manal vs. Vocal Task 2) & 1,46 & 32.15 & $<.001$ & .41 & 1,46 & 34.18 & $<.001$ & .43 \\
\hline SOA & $1.69,77.94$ & 441.67 & $<.001$ & .91 & $2.26,103.77$ & 478.43 & $<.001$ & .91 \\
\hline Group $\times$ SOA & $1.69,77.94$ & 9.24 & .001 & .17 & $2.26,103.77$ & 10.13 & $<.001$ & .18 \\
\hline
\end{tabular}

Open Access This article is licensed under a Creative Commons Attribution 4.0 International License, which permits use, sharing, adaptation, distribution and reproduction in any medium or format, as long as you give appropriate credit to the original author(s) and the source, provide a link to the Creative Commons licence, and indicate if changes were made. The images or other third party material in this article are included in the article's Creative Commons licence, unless indicated otherwise in a credit line to the material. If material is not included in the article's Creative Commons licence and your intended use is not permitted by statutory regulation or exceeds the permitted use, you will need to obtain permission directly from the copyright holder. To view a copy of this licence, visit http://creativecommons.org/licenses/by/4.0/.

\section{References}

Bratzke, D., Rolke, B., Ulrich, R., \& Peters, M. (2007). Central slowing during the night. Psychological Science, 18(5), 456-461. doi:https:// doi.org/10.1111/j.1467-9280.2007.01921.x

Bratzke, D., Ulrich, R., Rolke, B., Schröter, H., Jentzsch, I., \& Leuthold, H. (2008). Motor limitation in dual-task processing with different effectors. Quarterly Journal of Experimental Psychology, 61(9), 1385-1399. doi:https://doi.org/10.1080/17470210701536856

De Jong, R. (1995). The role of preparation in overlapping-task performance. The Quarterly Journal of Experimental Psychology Section, A, 48(1), 2-25. doi:https://doi.org/10.1080/14640749508401372

Durst, M., \& Janczyk, M. (2018). The motor locus of no-go backward crosstalk. Journal of Experimental Psychology: Learning, Memory, and Cognition, 44(12), 1931-1946. doi:https://doi.org/10.1037/xlm0000565

Durst, M., \& Janczyk, M. (2019). Two types of backward crosstalk: Sequential modulations and evidence from the diffusion model. Acta Psychologica, 193, 132-152. doi:https://doi.org/10.1016/j.actpsy.2018.11.013

Fagot, C., \& Pashler, H. (1992). Making two responses to a single object: Implications for the central attentional bottleneck. Journal of Experimental Psychology: Human Perception and Performance, 18(4), 1058-1079. doi:https://doi.org/10.1037/0096-1523.18.4.1058

Fischer, R., \& Plessow, F. (2015). Efficient multitasking: Parallel versus serial processing of multiple tasks. Frontiers in Psychology, 6, 1366. doi:https://doi.org/10.3389/fpsyg.2015.01366

Fischer, R., Gottschalk, C., \& Dreisbach, G. (2014). Context-sensitive adjustment of cognitive control in dual-task performance. Journal of Experimental Psychology: Learning, Memory, and Cognition, 40(2), 399-416. doi:https://doi.org/10.1037/a0034310

Hibberd, D. L., Jamson, S. L., \& Carsten, O. M. J. (2010). Managing invehicle distractions. In A. K. Dey, A. Schmidt, S. Boll, \& A. L. Kun (Eds.), Proceedings of the Second International Conference on Automotive User Interfaces and Interactive Vehicular Applications-Automotive UI '10 (4). New York, NY: ACM Press. doi:https://doi.org/10.1145/1969773.1969775
Hirsch, P., Declerck, M., \& Koch, I. (2015). Exploring the functional locus of language switching: Evidence from a PRP paradigm. Acta Psychologica, 161, 1-6. doi:https://doi.org/10.1016/j.actpsy.2015.07.010

Hirsch, P., Nolden, S., \& Koch, I. (2017). Higher-order cognitive control in dual tasks: Evidence from task-pair switching. Journal of Experimental Psychology: Human Perception and Performance, 43(3), 569-580. doi:https://doi.org/10.1037/xhp0000309

Hirsch, P., Nolden, S., Philipp, A. M., \& Koch, I. (2018). Hierarchical task organization in dual tasks: Evidence for higher level task representations. Psychological Research, 82(4), 759-770. doi:https:// doi.org/10.1007/s00426-017-0851-0

Hoffmann, M. A., Pieczykolan, A., Koch, I., \& Huestegge, L. (2019). Motor sources of dual-task interference: Evidence for effector-based prioritization in dual-task control. Journal of Experimental Psychology: Human Perception and Performance. 45(10), 13551374. doi:https://doi.org/10.1037/xhp0000677

Hoffmann, M. A., Westermann, M., Pieczykolan, A., \& Huestegge, L. (in press). Effects of input modality on vocal effector prioritization in manual-vocal dual tasks. Experimental Psychology.

Hommel, B. (1998). Automatic stimulus-response translation in dualtask performance. Journal of Experimental Psychology: Human Perception and Performance, 24(5), 1368-1384. doi:https://doi. org/10.1037//0096-1523.24.5.1368

Hommel, B., \& Eglau, B. (2002). Control of stimulus-response translation in dual-task performance. Psychological Research, 66(4), 260273. doi:https://doi.org/10.1007/s00426-002-0100-y

Huestegge, L. (2011). The role of saccades in multitasking: Towards an output-related view of eye movements. Psychological Research, 75(6), 452-465. doi:https://doi.org/10.1007/s00426-011-0352-5

Huestegge, L., \& Adam, J. J. (2011). Oculomotor interference during manual response preparation: Evidence from the response-cueing paradigm. Attention, Perception, \& Psychophysics, 73(3), 702707. doi:https://doi.org/10.3758/s13414-010-0051-0

Huestegge, L., \& Hazeltine, E. (2011). Crossmodal action: Modality matters. Psychological Research, 75(6), 445-451. doi:https://doi.org/ 10.1007/s00426-011-0373-0

Huestegge, L., \& Koch, I. (2010). Crossmodal action selection: Evidence from dual-task compatibility. Memory \& Cognition, 38(4), 493501. doi:https://doi.org/10.3758/MC.38.4.493

Huestegge, L., \& Koch, I. (2013). Constraints in task-set control: Modality dominance patterns among effector systems. Journal of Experimental Psychology. General, 142(3), 633-637. doi:https:// doi.org/10.1037/a0030156

Huestegge, L., Pieczykolan, A., \& Koch, I. (2014). Talking while looking: On the encapsulation of output system representations. Cognitive Psychology, 73, 72-91. doi:https://doi.org/10.1016/j.cogpsych.2014.06.001

Huestegge, L., Pieczykolan, A., \& Janczyk, M. (2018). Backward crosstalk and the role of dimensional overlap within and between tasks. Acta Psychologica, 188, 139-147. doi:https://doi.org/10.1016/j.actpsy.2018.06.004

Janczyk, M., \& Huestegge, L. (2017). Effects of a no-go Task 2 on Task 1 performance in dual-tasking: From benefits to costs. Attention, Perception, \& Psychophysics, 79(3), 796-806. doi:https://doi.org/ 10.3758/s13414-016-1257-6 
Janczyk, M., \& Kunde, W. (2010). Does dorsal processing require central capacity? More evidence from the PRP paradigm. Experimental Brain Research, 203(1), 89-100. doi:https://doi.org/10.1007/ s00221-010-2211-9

Janczyk, M., Augst, S., \& Kunde, W. (2014). The locus of the emotional Stroop effect: A study with the PRP paradigm. Acta Psychologica, 151, 8-15. doi:https://doi.org/10.1016/j.actpsy.2014.05.011

Janczyk, M., Pfister, R., Hommel, B., \& Kunde, W. (2014). Who is talking in backward crosstalk? Disentangling response- from goalconflict in dual-task performance. Cognition, 132(1), 30-43. doi: https://doi.org/10.1016/j.cognition.2014.03.001

Janczyk, M., Renas, S., \& Durst, M. (2018). Identifying the locus of compatibility-based backward crosstalk: Evidence from an extended PRP paradigm. Journal of Experimental Psychology: Human Perception and Performance, 44(2), 261-276. doi:https://doi.org/ 10.1037/xhp0000445

Koch, I., Poljac, E., Müller, H., \& Kiesel, A. (2018). Cognitive structure, flexibility, and plasticity in human multitasking: An integrative review of dual-task and task-switching research. Psychological Bulletin, 144(6), 557-583. doi:https://doi.org/10.1037/bul0000144

Kübler, S., Reimer, C. B., Strobach, T., \& Schubert, T. (2018). The impact of free-order and sequential-order instructions on task-order regulation in dual tasks. Psychological Research, 82(1), 40-53. doi: https://doi.org/10.1007/s00426-017-0910-6

Kunde, W., Wirth, R., \& Janczyk, M. (2018). The role of feedback delay in dual-task performance. Psychological Research, 82(1), 157-166. doi:https://doi.org/10.1007/s00426-017-0874-6

Künstler, E. C. S., Finke, K., Günther, A., Klingner, C., Witte, O., \& Bublak, P. (2018). Motor-cognitive dual-task performance: Effects of a concurrent motor task on distinct components of visual processing capacity. Psychological Research, 82(1), 177-185. doi:https:// doi.org/10.1007/s00426-017-0951-x

Lehle, C., \& Hübner, R. (2009). Strategic capacity sharing between two tasks: Evidence from tasks with the same and with different task sets. Psychological Research, 73(5), 707-726. doi:https://doi.org/ 10.1007/s00426-008-0162-6

Logan, G. D., \& Gordon, R. D. (2001). Executive control of visual attention in dual-task situations. Psychological Review, 108(2), 393-434. doi:https://doi.org/10.1037/0033-295X.108.2.393

Luria, R., \& Meiran, N. (2003). Online order control in the psychological refractory period paradigm. Journal of Experimental Psychology: Human Perception and Performance, 29(3), 556-574. doi:https:// doi.org/10.1037/0096-1523.29.3.556

Meyer, D. E., \& Kieras, D. E. (1997). A computational theory of executive cognitive processes and multiple-task performance: Part 2. Accounts of psychological refractory-period phenomena. Psychological Review, 104(4), 749-791. doi:https://doi.org/10.1037/0033-295X.104.4.749

Miller, J., Ulrich, R., \& Rolke, B. (2009). On the optimality of serial and parallel processing in the psychological refractory period paradigm: Effects of the distribution of stimulus onset asynchronies. Cognitive Psychology, 58(3), 273-310. doi:https://doi.org/10.1016/j.cogpsych.2006.08.003

Navon, D., \& Miller, J. (2002). Queuing or sharing? A critical evaluation of the single-bottleneck notion. Cognitive Psychology, 44(3), 193251. doi:https://doi.org/10.1006/cogp.2001.0767

Pashler, H. (1984). Processing stages in overlapping tasks: Evidence for a central bottleneck. Journal of Experimental Psychology: Human Perception and Performance, 10(3), 358-377. doi:https://doi.org/ 10.1037/0096-1523.10.3.358

Pashler, H. (1994). Dual-task interference in simple tasks: Data and theory. Psychological Bulletin, 116(2), 220-244. doi:https://doi.org/10. 1037/0033-2909.116.2.220

Pashler, H., Carrier, M., \& Hoffman, J. (1993). Saccadic eye movements and dual-task interference. The Quarterly Journal of Experimental Psychology Section A, 46(1), 51-82. doi:https://doi.org/10.1080/ 14640749308401067
Peterson, L. R. (1969). Concurrent verbal activity. Psychological Review, 76(4), 376-386. doi:https://doi.org/10.1037/h0027443

Pfister, R., \& Kunde, W. (2013). Dissecting the response in responseeffect compatibility. Experimental Brain Research, 224(4), 647655. doi:https://doi.org/10.1007/s00221-012-3343-x

Pfister, R., Janczyk, M., Gressmann, M., Fournier, L. R., \& Kunde, W. (2014). Good vibrations? Vibrotactile self-stimulation reveals anticipation of bodyrelated action effects in motor control. Experimental Brain Research, 232(3), 847-854. doi:https://doi.org/10.1007/s00221-013-3796-6

Pieczykolan, A., \& Huestegge, L. (2014). Oculomotor dominance in multitasking: Mechanisms of conflict resolution in cross-modal action. Journal of Vision, 14(13), 18. doi:https://doi.org/10.1167/14.13.18

Pieczykolan, A., \& Huestegge, L. (2017). Cross-modal Action Complexity: Action- and Rule-related Memory Retrieval in Dualresponse Control. Frontiers in Psychology, 8, 529. doi:https://doi. org/10.3389/fpsyg.2017.00529

Pieczykolan, A., \& Huestegge, L. (2018). Sources of interference in cross-modal action: Response selection, crosstalk, and general dual-execution costs. Psychological Research, 82(1), 109-120. doi:https://doi.org/10.1007/s00426-017-0923-1

Pieczykolan, A., \& Huestegge, L. (2019). Action scheduling in multitasking: A multi-phase framework of response-order control. Attention, Perception \& Psychophysics. Advance online publication. doi: https://doi.org/10.3758/s13414-018-01660-w

Renas, S., Durst, M., \& Janczyk, M. (2018). Action effect features, but not anatomical features, determine the backward crosstalk effect: Evidence from crossed-hands experiments. Psychological Research, 82(5), 970 980. doi:https://doi.org/10.1007/s00426-017-0873-7

Schumacher, E. H., Seymour, T. L., Glass, J. M., Fencsik, D. E., Lauber, E. J., Kieras, D. E., \& Meyer, D. E. (2001). Virtually perfect time sharing in dual-task performance: Uncorking the central cognitive bottleneck. Psychological Science, 12(2), 101-108. doi:https://doi. org/10.1111/1467-9280.00318

Schweickert, R. (1978). A critical path generalization of the additive factor method: Analysis of a Stroop task. Journal of Mathematical Psychology, 18(2), 105-139. doi:https://doi.org/10.1016/0022-2496(78)90059-7

Spelke, E., Hirst, W., \& Neisser, U. (1976). Skills of divided attention. Cognition, 4(3), 215-230. doi:https://doi.org/10.1016/0010-0277(76)90018-4

Stelzel, C., Schumacher, E. H., Schubert, T., \& D'Esposito, M. (2006). The neural effect of stimulus-response modality compatibility on dual-task performance: An fMRI study. Psychological Research, 70(6), 514-525. doi:https://doi.org/10.1007/s00426-005-0013-7

Strobach, T., Becker, M., Schubert, T., \& Kühn, S. (2015). Better dualtask processing in simultaneous interpreters. Frontiers in Psychology, 6, 1-9. doi:https://doi.org/10.3389/fpsyg.2015.01590

Strobach, T., Kübler, S., \& Schubert, T. (2019) Endogenous control of task-order preparation in variable dual tasks. Psychological Research, 1-19. doi:https://doi.org/10.1007/s00426-019-01259-2

Telford, C. W. (1931). The refractory phase of voluntary and associative responses. Journal of Experimental Psychology, 14(1), 1-36. doi: https://doi.org/10.1037/h0073262

Tombu, M., \& Jolicœur, P. (2003). A central capacity sharing model of dual-task performance. Journal of Experimental Psychology: Human Perception and Performance, 29(1), 3-18. doi:https://doi. org/10.1037/0096-1523.29.1.3

Welford, A. T. (1952). The 'psychological refractory period' and the timing of high-speed performance - A review and a theory. British Journal of Psychology. General Section, 43(1), 2-19. doi:https:// doi.org/10.1111/j.2044-8295.1952.tb00322.x

This study was not preregistered, but the planned procedure was described in detail in the DFG funding proposal (Project HU 1847/7-1; see https://gepris.dfg.de/gepris/projekt/274340856 for a description of the project). 